\title{
Downregulation of tetrahydrobiopterin inhibits tumor angiogenesis in BALB/c-nu mice with hepatocellular carcinoma
}

\author{
YOUGUO DAI ${ }^{1}$, JIN CUI $^{2}$, PING GAN ${ }^{1}$ and WEIMING $\mathrm{LI}^{3}$ \\ ${ }^{1}$ Department of Abdominal Surgery, Third Affiliated Hospital of Kunming Medical University, \\ Kunming, Yunnan 650118; Departments of ${ }^{2}$ Pathology and ${ }^{3}$ General Surgery, \\ Second Affiliated Hospital of Kunming Medical University, Kunming, Yunnan 650101, P.R. China
}

Received October 25, 2015; Accepted December 4, 2015

DOI: $10.3892 /$ or.2016.4850

\begin{abstract}
Hepatocellular carcinoma (HCC) is a highly vascular tumor, and treatment options for patients of advanced-stage are limited. Nitric oxide (NO), which is derived from endothelial nitric oxide synthase (eNOS), provides crucial signals for angiogenesis in the tumor microenvironment. Tetrahydrobiopterin (BH4) is an essential cofactor eNOS and represents a critical determinant of $\mathrm{NO}$ production. To examine whether treatment of 2,4-diamino-6-hydroxypyrimidine (DAHP) inhibits angiogenesis of HCC, BALB/c-nu mice were injected with HepG-2 cells with DAHP. Supplemental DAHP treatment decreased K-ras mRNA transcripts, inhibition of phosphorylation of eNOS and Akt, inhibition of guanosine triphosphate cyclohydrolase (GTPCH), and decreased significantly NO synthesis, and then inhibited angiogenesis, compared with the results observed in the saline group. Histopathology demonstrated angiogenesis and tumor formation were significantly inhibited in HCC. DAHP downregulates GTPCH protein expression, corresponding to decreased levels of $\mathrm{BH} 4$ and the contents of NO. In addition, DAHP downregulates eNOS and Akt protein expression, corresponding to decreased eNOS phosphorylation at Ser1177 and Akt phosphorylation, compared with the saline control. We suggest that DAHP, recognized as a specific competitive inhibitor of GTPCH, can decrease tumor BH4 and NO by the inhibition of the wild-type Ras-PI3K/Akt pathway, and then inhibiting angiogenesis, and may provide a novel and promising way to target $\mathrm{BH} 4$ synthetic pathways to inhibit angiogenesis and to control potential progression of HCC. Whether DAHP has a therapeutic potential will require more direct testing in humans.
\end{abstract}

Correspondence to: Dr Youguo Dai, Department of Abdominal Surgery, Third Affiliated Hospital of Kunming Medical University, Kunming, Yunnan 650118, P.R. China

E-mail: daiyouguo@126.com

Keywords: tetrahydrobiopterin, hepatocellularcarcinoma, nitric oxide, endothelial nitric oxide synthase, 2,4-diamino-6-hydroxypyrimidine

\section{Introduction}

A process of generation of new blood vessels has been proved to be necessary for sustained tumor growth and cancer progression. Inhibiting angiogenesis pathway has long been a significant hope for the development of novel, effective and target orientated antitumor agents arresting the tumor proliferation and metastasis (1).

Nitric oxide (NO) is a free radical involved in physiological as well as in pathophysiological processes, and synthesized from the conversion of L-arginine to citrulline by three distinct forms of NO synthase (NOS): endothelial NOS (eNOS), inducible NOS and neuronal NOS in different cell types and tissues (2).

One of the many physiological functions of $\mathrm{NO}$ is as an important modulator of endothelial function pertaining to angiogenesis (3). NO has been shown to stimulate and inhibit the proliferation, migration and differentiation of endothelial cells in vitro and angiogenesis in vivo (4). In tumor biology, NO was demonstrated to promote either tumor invasion and angiogenesis or tumor regression (5), chiefly increasing DNA synthesis, cell proliferation and migration of endothelial cells to promote tumor angiogenesis (6).

Specifically, eNOS was shown to modulate cancer-related events (angiogenesis, apoptosis, cell cycle, invasion and metastasis) and genetic studies showed that eNOS gene polymorphisms are associated with the development of multiple types of cancers $(7,8)$. The present study found a positive correlation between the expression of nitric oxide synthase (NOS) and tumor progression; treatment with inhibitors of NOS, NG-methyl-1-arginine (NMMA) and NG-nitro-l-arginine methyl ester (L-NAME), had antitumor and antimetastatic effects that were partly attributed to reduced tumor cell invasiveness (9). The above suggests that eNOS-derived NO signaling is one of the key factors in regulating endothelial function and inducing tumor angiogenesis.

Tetrahydrobiopterin (BH4) is an essential cofactor required for the activity of eNOS. Suboptimal concentrations of BH4 in the endothelium reduce the biosynthesis of NO, and preferentially produces superoxide (10) and contributes to angiogenesis regulation (11). In vivo, de novo $\mathrm{BH} 4$ biosynthesis is regulated 
by the ratelimiting enzyme guanosine triphosphate cyclohydrolase I (GTPCH; EC 3.5.4.16), which converts GTP to dihydroneopterin triphosphate. BH4 is produced through further steps, which are catalyzed by 6-pyruvoyl tetrahydropterin synthase and sepiapterin reductase (12). Recent evidence suggests an important role for $\mathrm{BH} 4$ synthesis in angiogenesis by the activation of eNOS for NO production, which is maintained by a phosphatidylinositol 3-kinase (PI3K)/Akt positive feedback loop through effects on wild-type Ras in endothelial cells (13). Theoretically, BH4 biosynthesis has been directly associated with activation of Akt and eNOS phosphorylation in inducing angiogenesis (14). However, impacts of downregulation of endogenous BH4 by GTPCH pathway on angiogenesis and the mechanism is not known in hepatocellular carcinoma (HCC).

In the present study, we investigated the effects of downregulation of $\mathrm{BH} 4$ by 2,4-diamino-6-hydroxypyrimidine (DAHP) on anti-angiogenesis and explored the mechanism via a BALB/c-nu mouse HCC xenograft model. On the basis of our results, we propose that downregulation of $\mathrm{BH} 4$ could be a promising novel therapeutic strategy, and GTPCH could be a new target of the treatment for HCC.

\section{Materials and methods}

Reagents. 6R-5,6,7,8-Tetrahydrobiopterin dihydrochloride (BH4) and DAHP were purchased from Sigma-Aldrich (Shanghai, China), and dissolved in phosphate-buffered saline (PBS).

Cell culture. Primary human umbilical vein endothelial cells (HUVECs) were purchased from American Type Culture Collection (ATCC; Manassas, VA, USA) and grown in endothelial cell growth medium-2 (EGM-2) with bullet kit at $37^{\circ} \mathrm{C}$ in $5 \% \mathrm{CO}_{2}$. HUVECs were used at passages 4-10. Before experiments, the cells were deprived of fetal bovine serum (FBS) for 3-4 $\mathrm{h}$ for addition of reagents. All samples were determined in triplicate.

HepG-2 cells purchased from the Institute of Yunnan Provincial Tumors (Kunming, China) and grown in RPMI-1640 with $10 \% \mathrm{FBS}$ and with bullet kit at $37^{\circ} \mathrm{C}$ in $5 \% \mathrm{CO}_{2}$ in our laboratory. All samples were determined in triplicate.

Tubulogenesis. Ninety-six-well culture plates were coated with $50 \mu \mathrm{l} /$ well of growth factor-reduced Matrigel (BD Biosciences, Shanghai, China). HUVECs (1×10\%/well) were incubated with $2 \% \mathrm{FBS}$ for $24 \mathrm{~h}$. Then, the cells were incubated with $30 \mu \mathrm{g} /$ well of DAHP for $48 \mathrm{~h}$. The number of loops was counted in images captured at a magnification of $\mathrm{x} 20$.

Animals. BALB/c-nu mice used in the present study were obtained from Vital River Laboratory Animal Technology Co. Ltd. (Beijing, China) and were maintained on a standard $12 \mathrm{~h}: 12 \mathrm{~h}$ light-dark cycle with free access to food and water. Male mice, aged 4-6 weeks and weighing 18-22 g were used in the experiments. All of the animal procedures were in accordance with the institutional guidelines of Kunming Medical University.

Animal procedures and experimental groups. In the axillary region, mice received subcutaneous implants of $1 \times 10^{7}$ HepG2 cells. Cells $\left(10^{7}\right)$ suspended in $100 \mu$ of PBS (BD Biosciences) were subcutaneously injected into flanks of BALB/c-nu mice and resultant tumors measured twice weekly. Mice were treated with $80 \mathrm{mg} / \mathrm{kg}$ (i.p.) DAHP once a day for two weeks once tumors reached a volume of $100 \mathrm{~mm}^{3}$ as previously described (15). Equal volumes of saline were injected in control mice. The long and short diameter of tumor was, respectively, recorded as A and B two weeks after the treatment, and then the volume of the tumor was calculated via the formula $\mathrm{V}=0.5 \times \mathrm{A} \times \mathrm{B}^{2}$.

Histopathology. Samples were respectively harvested after a period of time. The tumor were fixed using $4 \%$ formaldehyde and were embedded using paraffin then serially sectioned (5 mm) in toto. Every third slide was stained with hematoxylin and eosin (H\&E) for histomorphometric analyses. One or two images/slide were captured with a microscope (Olympus, Japan) at a magnification of $\mathrm{x} 200$ and at a resolution of $640 \times 480$ pixels.

Immunohistochemistry for CD31. Paraffin-embedded tissue blocks from formalin-fixed tumor samples were sectioned, dewaxed and rehydrated following standard protocols. Sections were incubated for $2 \mathrm{~h}$ at room temperature with the anti-CD31 monoclonal antibody (Proteintech, Wuhan, China), followed by incubations with saturating amounts of biotin-labeled secondary antibody and streptavidin-peroxidase for $20 \mathrm{~min}$ each. After incubation in a solution containing $0.06 \mathrm{mM}$ diaminobenzidine (Dako) and $2 \mathrm{mM}$ hydrogen peroxide in $0.05 \%$ PBS ( $\mathrm{pH} 7.6$ ) for $5 \mathrm{~min}$, slides were washed, dehydrated with alcohol and xylene, and mounted with coverslips. Sections were examined with a microscope (Olympus) and images were acquired at a magnification of $x 400$. For quantification, the product of the proportion of positive cells in quartiles (0-4) and the staining intensity ( 0 , no staining; 1 , weak; 2 , moderate; and 3 , strong) was calculated, yielding a total immunostaining score ranging from 0 to 12 .

Western blotting. Thirty micrograms of cytosolic protein was electrophoresed on $12 \%$ acrylamide sodium dodecyl sulfate gels and was transferred to nitrocellulose membranes (Schleicher \& Schuell, Keene, NH, USA). To block non-specific binding, $5 \%$ non-fat dry milk in PBS-Tween $(0.1 \%)$ was added to the membrane for $1 \mathrm{~h}$ at room temperature. Membranes were washed in PBS-Tween then incubated overnight with mouse mAbs for eNOS, p-eNOS, AktT, p-AkT and GTPCH (dilution 1:1,000; Santa Cruz Biotechnology, Shanghai, China), and a rabbit anti-mouse polyclonal antibody for mouse $\beta$-actin (dilution 1:100; Kangwei Shiji Biotechnology, Peking, China) followed by incubation with a secondary antibody for $1 \mathrm{~h}$. After repeat washings with PBS-Tween, membranes were developed using the SuperSignal detection system (Pierce Chemical, Rockford, IL, USA) and were exposed to film. Quantification of the western blot analyses for eNOS, p-eNOS, Akt, p-Akt and GTPCH was performed using gel analysis software.

Quantitative real-time RT-PCR for K-ras $m R N A$. Total RNA was extracted from frozen tumor tissue by homogenization in $1 \mathrm{ml}$ of TRIzol solution (Sigma-Aldrich). The tumor was incubated for $5 \mathrm{~min}$ in $1 \mathrm{ml}$ of TRIzol, and the residual tissue was 

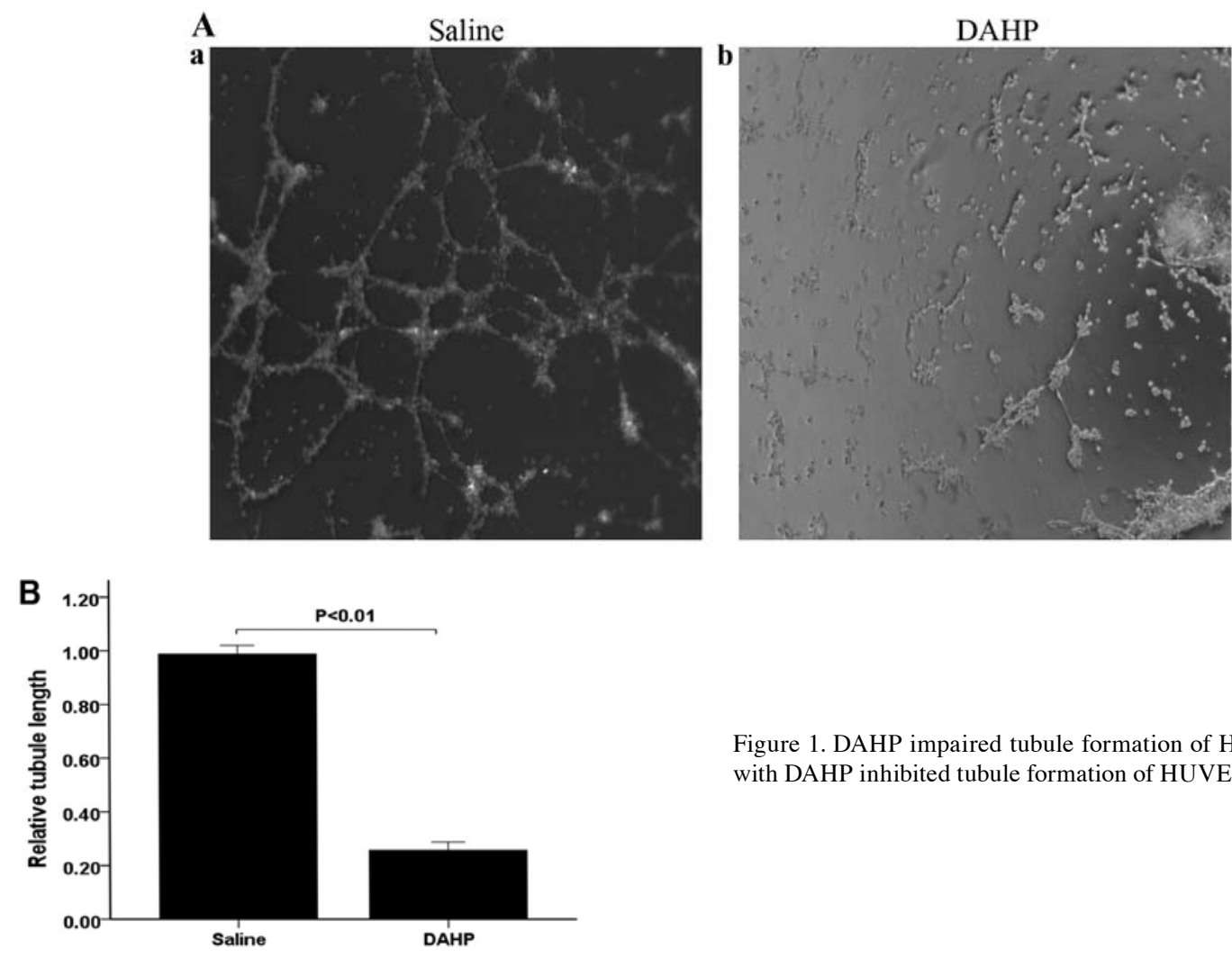

Figure 1. DAHP impaired tubule formation of HUVEC in vitro. Treatment with DAHP inhibited tubule formation of HUVEC.

removed. Total tissue RNA (1 $\mu \mathrm{g})$, dissolved in RNase-free water was used in RT-PCR (Maxima ${ }^{\circledR}$ QuantiTect SYBRGreen RT-PCR kit; Fermentas, Shanghai, China) using the following primers for various K-ras mRNA transcripts (5'-3'): mouse K-ras forward, TGCAAACTGTCAGCTTTATCTCAA and reverse, CTTCATTATCCTGCTTCCCATC; mouse $\beta$-actin forward, CGTGCGTGACATCAAAGAGAAG and reverse, CCAAGAAGGAAGGCTGGAAAA. Quantitative fluorescent real-time RT-PCR analysis was performed to compare relative quantities of mRNA in mouse tumor tissue using the ABI PRISM 7300 system (Applied Biosystems Inc., Foster City, CA, USA). Samples were processed in triplicate, with a reverse transcriptase (RT) negative control reaction for each sample. For K-ras RT-PCR analysis, standards were prepared using 10-fold dilutions of BALB/c-nu/nu mouse tumor total RNA. Quantification was performed using DataAssist $^{\mathrm{TM}}$ v3.0 software (Applied Biosystems Inc.) to generate standard curves, expressing relative quantities of PCR products in the experimental samples in arbitrary units relative to the standard curve. Mean values were calculated from triplicate samples to produce 1. Results from at least six animals per group were pooled to produce the mean and SEM.

NO content. Tumor tissue nitrite and nitrate levels were determined using a commercially available kit (Beyotime Biotechnology, Shanghai, China).

Biopterin measurements. Total biopterin (BH4, BH2 and biopterin) and $\mathrm{BH} 4$ (BH4=total biopterin, $\mathrm{BH} 2$-biopterin) levels were measured using high-performance liquid chromatography (HPLC) analysis with fluorescent detection after differential iodine oxidation of tissue extracts in either acidic or alkaline conditions as previously described (16).

Statistical analysis. The data are expressed as the mean \pm SEM. Statistical significance of differences between means was assessed using independent sample t-test. SPSS for Windows version 18.0 (SPSS, Inc., Chicago, IL, USA) was used to complete all of the analyses, and a P-value of $<0.05$ was considered to indicate a statistically significant result.

\section{Results}

Effects of the administration of DAHP on tubulogenesis of $H U V E C$ in vitro. We firstly determined the effects of the administration of DAHP on tubule formation of HUVEC in vitro. The relative tubule length was significantly less in the DAHP group $(0.99 \pm 0.03)$ than in the saline group $(0.26 \pm 0.03$; $\mathrm{P}<0.01$ ) (Fig. 1). This confirms that the administered DAHP inhibits tubule formation of HUVEC in vitro.

Effects of the administration of DAHP on BH4 levels in the tumor tissue. We next determined whether the administration of DAHP resulted in downregulation of $\mathrm{BH} 4$ in tumor tissues. Total tumor biopterin and BH4 tissue levels were assessed by HPLC. Values are expressed as mean \pm SEM ( $\mathrm{n}=6$ animals/group) in $\mu \mathrm{g} / \mathrm{ml}$. BH4 levels was significantly lower in the DAHP group (BH4: $0.12 \pm 0.01$ ) than in the saline group (BH4:0.17 $\pm 0.01 ; \mathrm{P}<0.01)$ (Fig. $2 \mathrm{~A}$ ). This confirms that the administered DAHP decreases BH4 levels of the tumor tissue.

Effects of the administration of DAHP on NO production. NO content was assessed using tumor tissue nitrite/nitrate levels as indirect parameters. Nitrite/nitrate levels in the tumor tissue were determined by the Greiss reaction. Nitrite/nitrate levels in the tumor tissue were significantly decreased by $\sim 2$-fold in 

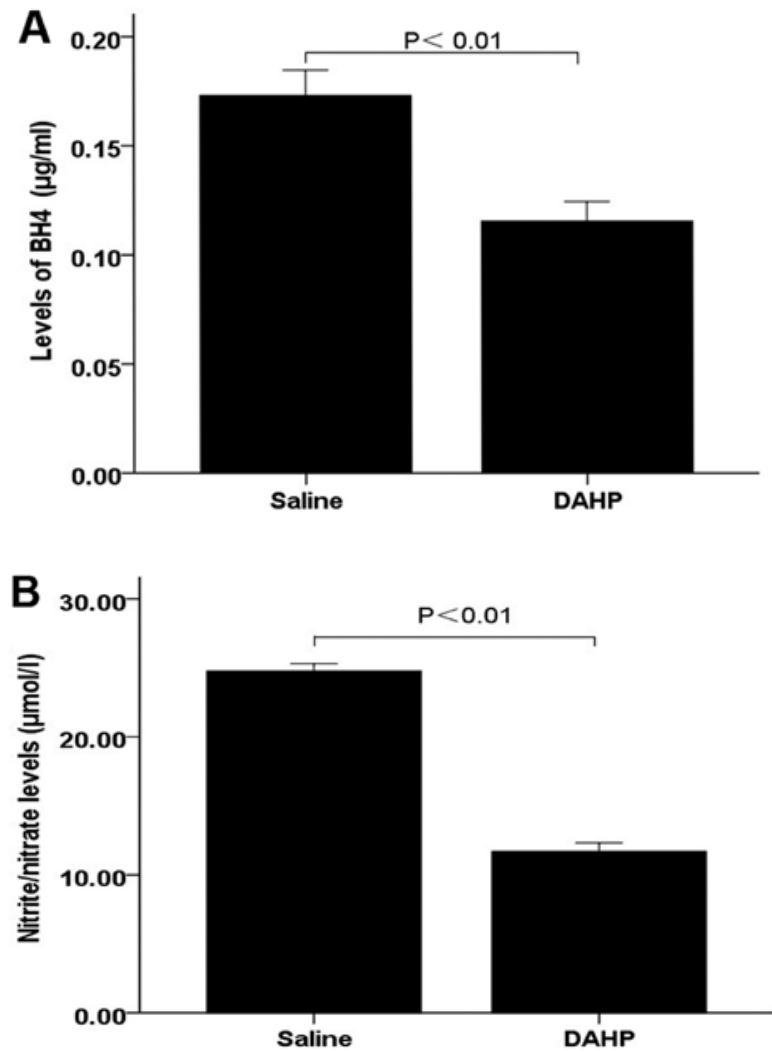

Figure 2. DAHP modulates BH4 levels and NO content in tumor tissue. (A) Total tumor biopterin and BH4 tissue levels were assessed by HPLC The administered DAHP decreases BH4 levels of tumor tissue in HCC. (B) Nitrite/nitrate levels in the tumor tissue was determined by the Greiss reaction. These levels indirectly measure NO content. The administration of DAHP significantly decreased nitrite/nitrate levels in tumor tissue.

the DAHP group (nitrite/nitrate: $11.70 \pm 0.62$ ) compared with that observed in the saline group (nitrite/nitrate: $24.77 \pm 0.54$; $\mathrm{P}<0.01$ ) (Fig. 2B). These findings suggest that treatment with DAHP decreases NO synthesis of the tumor tissue.

Morphology and histopathology. The tumor volume was significantly lower in the DAHP group $(122.87 \pm 5.39)$ than in the saline group (325.45 \pm 7.25 ; $\mathrm{P}<0.01)$ (Fig. 3A). Histological findings determined by $\mathrm{H} \& \mathrm{E}$ staining were quantified using Image Pro Plus software. Treatment with DAHP (449.20 \pm 43.46$)$ led to a significantly decreased number of cancer cells compared, respectively, with the saline group $(1,171.20 \pm 51.67$; $\mathrm{P}<0.01$ ) (Fig. 3B). These findings suggest that treatment with DAHP inhibits growth of tumors in HCC.

Effects of the administration of DAHP on the expression of CD31 protein. Tumor angiogenesis was assessed by CD31 immunostaining. CD31 in the tumor tissue was significantly lower in the DAHP group $(1.40 \pm 0.11)$ compared with that observed in the saline group $(1.94 \pm 0.15 ; \mathrm{P}<0.01)$ (Fig. 4). These findings suggest that treatment with DAHP inhibits tumor angiogenesis.

Effects of the administration of DAHP on the activation of wild-type Ras protein. We next invetigated the effects of downregulation of BH4 levels on K-ras mRNA by comparing with the saline control. K-ras mRNA in the tumor tissue was
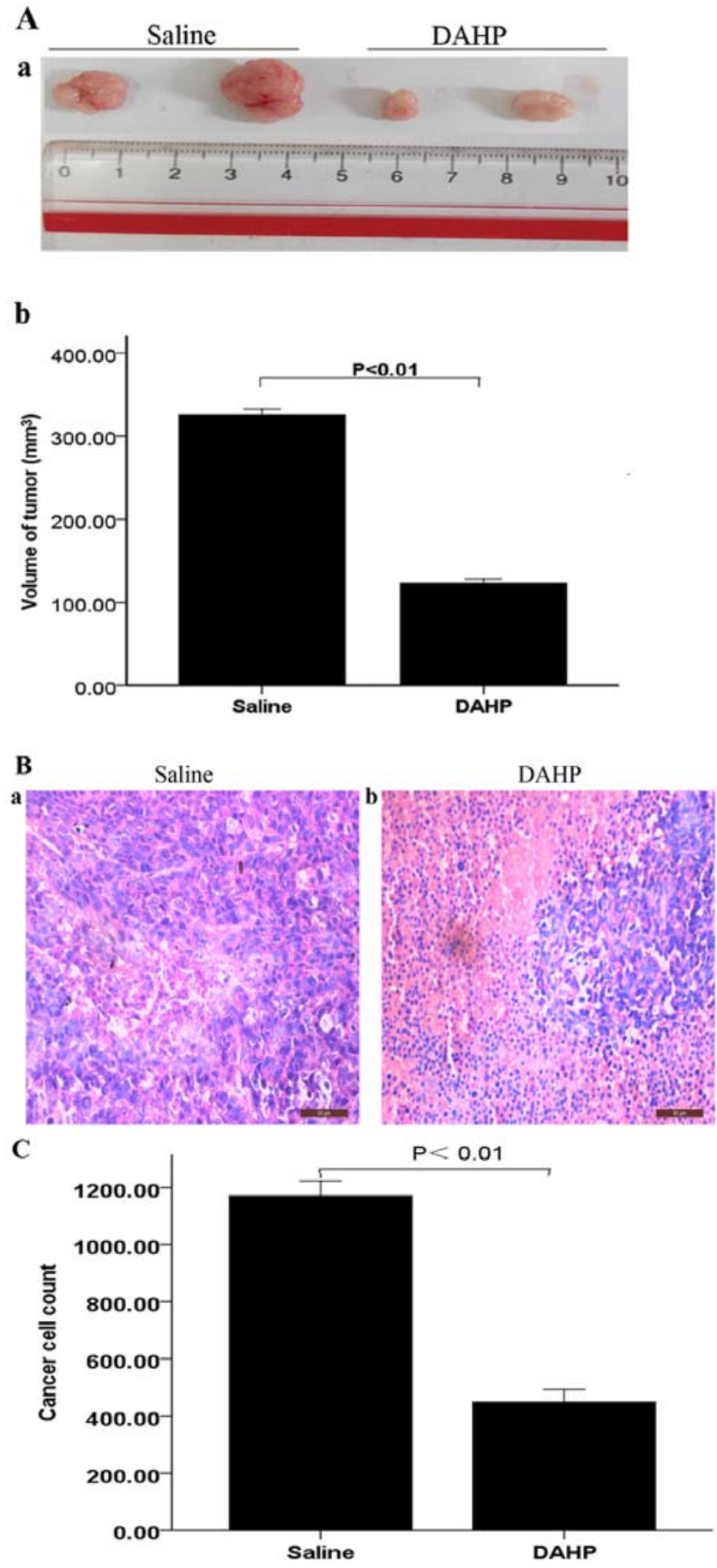

Figure 3. DAHP inhibits tumor formation in BALB/c-nu mice. (A) Volume of tumor significantly decreased in DAHP group compared with the saline group. (B) Treatment with DAHP inhibited formation of the tumor.

significantly lower in the DAHP group $(0.99 \pm 0.10)$ compared with the saline group $(3.14 \pm 0.13 ; \mathrm{P}<0.01)$ (Fig. 5A). These findings suggest that treatment with DAHP decreases K-ras mRNA of the tumor.

Effects of the administration of DAHP on GTPCH expression in angiogenesis. We investigated the effects of DAHP specifically on the expression of GTPCH by comparing saline-treated mice. GTPCH in the tumor tissue was significantly lower in the DAHP group $(0.78 \pm 0.04)$ compared with the saline group $(0.86 \pm 0.06 ; \mathrm{P}<0.05)$ (Fig. $5 \mathrm{~B})$. These 
A
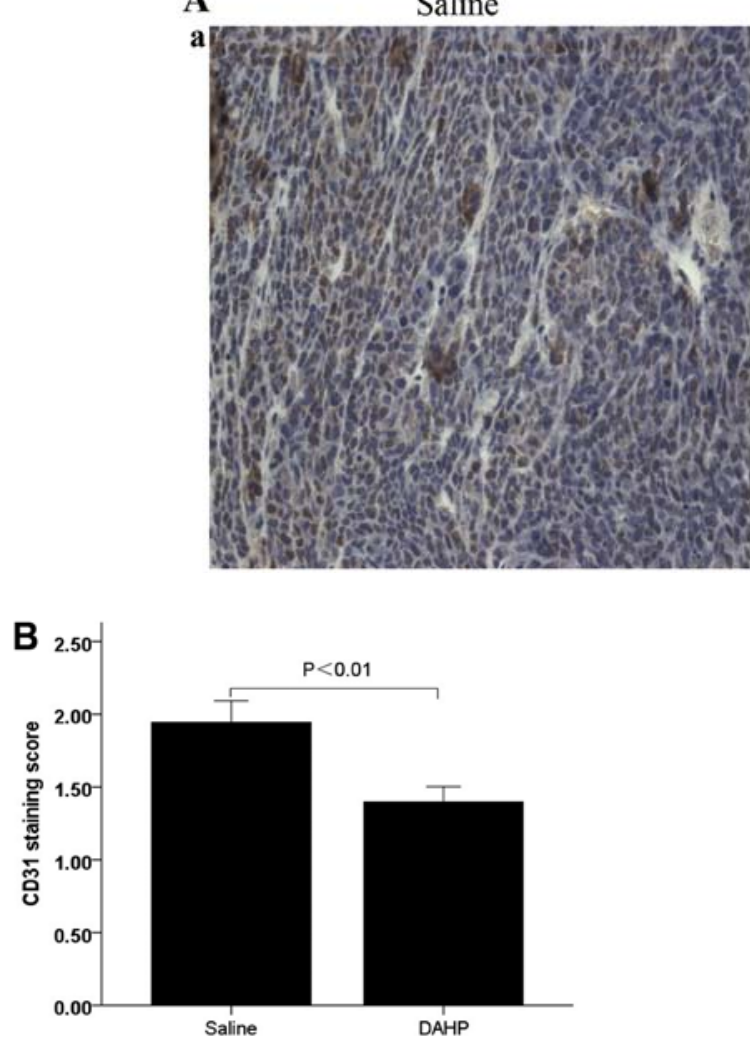

findings suggest that treatment with DAHP decreases the expression of GTPCH, with a corresponding decrease in the BH4 levels.

Effects of the administration of DAHP on phosphorylation of eNOS and Akt via PI3K pathway. We next investigated the effects of downregulation of $\mathrm{BH} 4$ levels specifically on phosphorylation of eNOS and Akt via PI3K pathway by comparing saline-treated mice. Expression of p-eNOS (Ser ${ }^{1177}$ ) and $\mathrm{p}-\mathrm{Akt}\left(\mathrm{Ser}^{473}\right)$ in the tumor tissue were significantly lower in the administration with DAHP group compared with that observed in the saline group (p-eNOS: $0.60 \pm 0.03$ vs. saline: $0.87 \pm 0.06$; $\mathrm{P}<0.01$; p-Akt: $0.67 \pm 0.03$ vs. saline: $0.91 \pm 0.05$; $\mathrm{P}<0.01$ ) (Fig. 6).

These results showed that DAHP inhibits PI3K-dependent phosphorylation of Akt/eNOS, with a corresponding decrease in the angiogenesis markers such as CD31. Moreover, the consequent descension of $\mathrm{NO}$ is paralleled by inhibition of intracellular wild-type Ras and PI3K signaling.

\section{Discussion}

There are many treatment options for patients with early-stage hepatocellular carcinoma (HCC), but the treatment options in advanced-stage HCC are limited, and the survival rate is dismal. $\mathrm{HCC}$ is a highly vascular tumor, its growth is dependent on the formation of new blood vessels (17). Anti-angiogenic therapies with sorafenib were the first systemic therapy to demonstrate improved survival in patients with advanced-stage HCC (18). This important development in the treatment of HCC raises hope, but cannot meet the needs of the patients. Thus, novel therapeutic approaches are desperately needed.

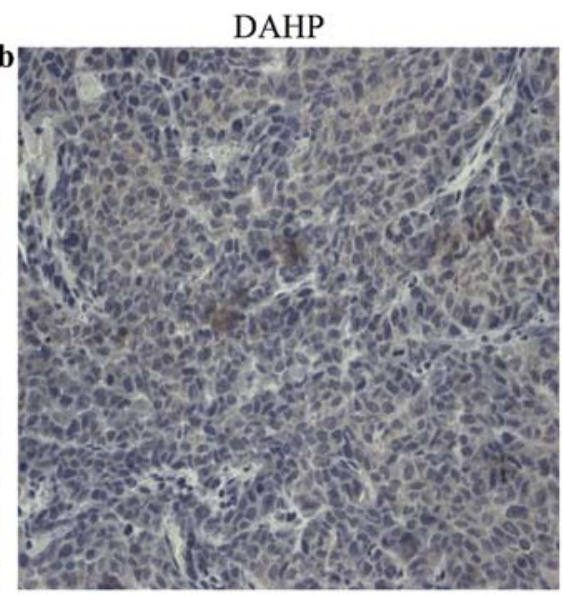

Figure 4. DAHP affects intratumoral expression of angiogenesis marker CD31 CD31 staining score for endothelial cells was notably lower in DAHP groups compared to the saline group, indicating less angiogenic activity, which is consistent with the downregulation of K-ras, GTPCH, p-eNOS and p-Akt.

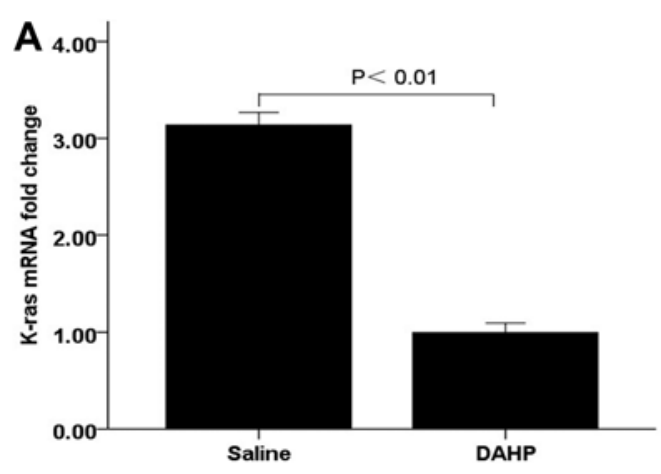

B
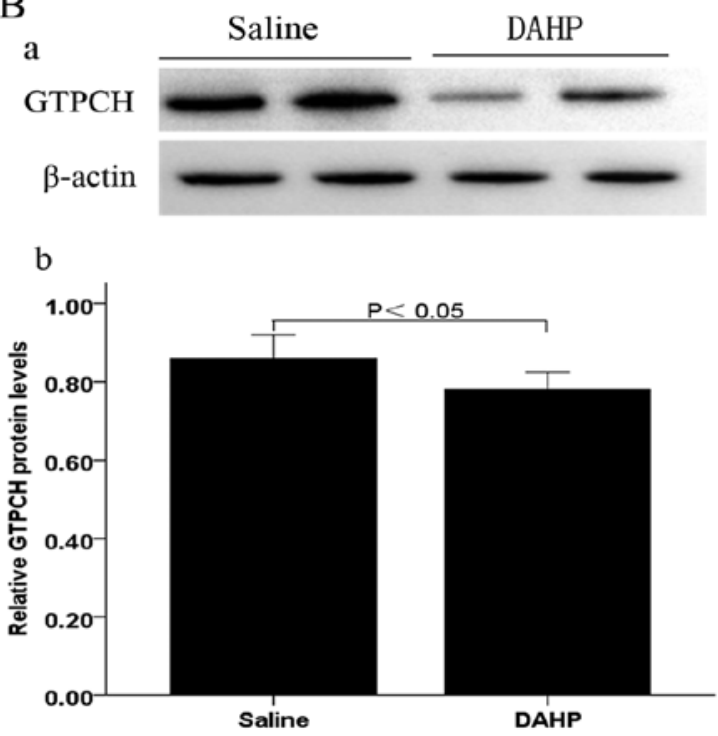

Figure 5. DAHP inhibits the expression of K-ras mRNA and GTPCH in tumor tissue. (A) K-ras mRNA in tumor tissue was measured by quantitative real-time RT-PCR. Treatment with DAHP decreased K-ras mRNA of the tumor. (B) Lysates of tumor tissue were fractionated by SDS-PAGE and immunoblotted with monoclonal antibody to GTPCH. 
A

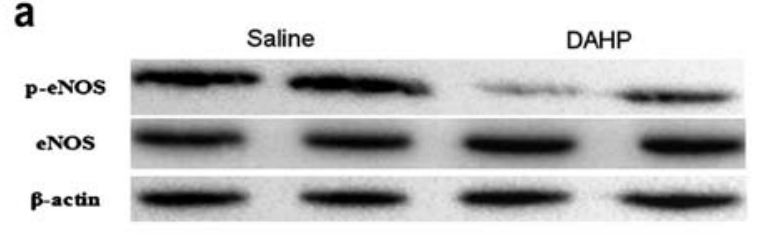

b

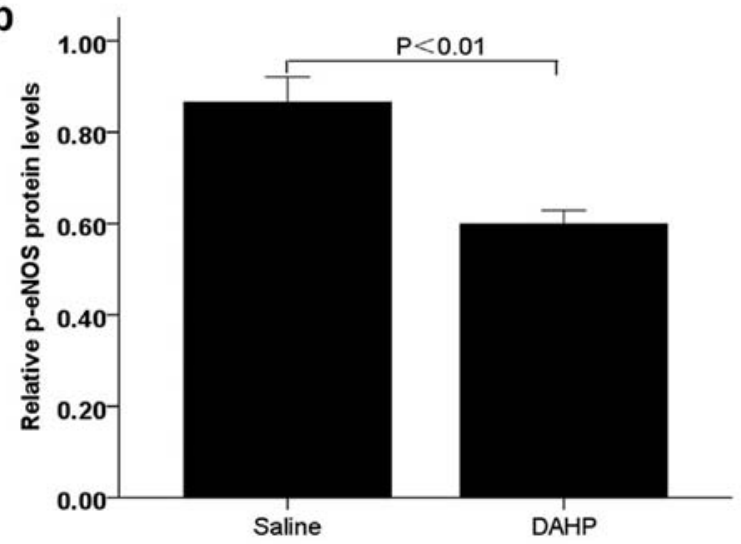

B a

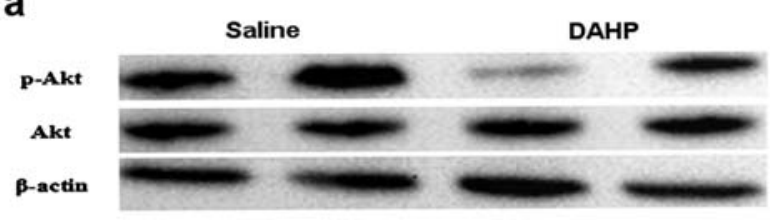

b

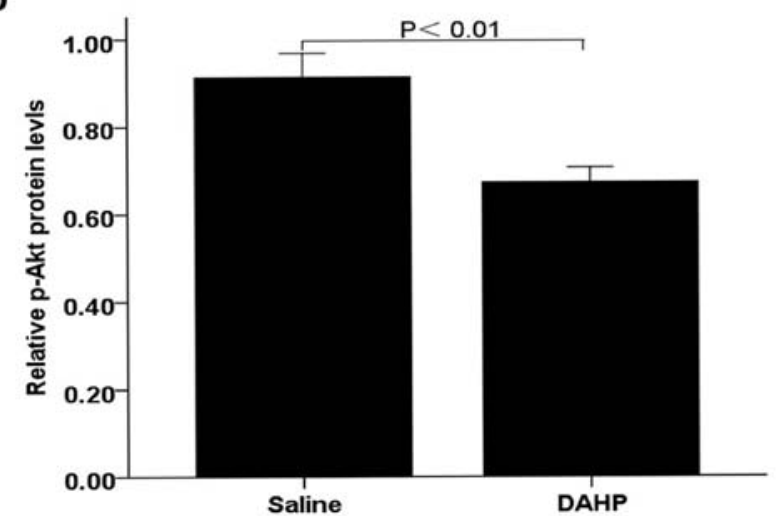

Figure 6. DAHP inhibits the phosphorylation of eNOS and Akt by downregulation of BH4 synthesis depending on PI3K signaling in tumor tissue. Lysates of tumor tissue were fractionated by SDS-PAGE and immunoblotted with monoclonal antibody to p-eNOS (Ser1177) and p-Akt (Ser473). Band intensity of p-eNOS and p-Akt were quantified on Quantity One 4.6 software. Treatment with DAHP inhibited the expression of p-eNOS and p-Akt in the tumor.

As previously described, multiple Ras family members directly bind to and activate the p110 catalytic subunit of the class I PI3Ks (19). Ras driven tumors exploit the functions of PI3K pathways in mitosis, apoptosis, motility, proliferation and differentiation. PI3K and Akt may regulate tumor angiogenesis by the induction of NOS (20). Tumor angiogenesis is regulated by the tumor microenvironment composed of tumor, vascular endothelial and stromal cells. In addition to cancer cells, the microvascular endothelial cells recruited by the tumor are important for cancer development $(21,22)$. PI3K/Akt pathway also controls the tumor microenvironment, including endothelial cells (23). PI3K can regulate endothelial migration, proliferation and survival through the effect of its downstream targets such as NOS, to regulate tumor angiogenesis (24). Our data suggested that inhibiting $\mathrm{BH} 4$ synthesis can decrease K-ras mRNA, and activation of phosphorylation of eNOS and Akt, and inhibited the produces of NO. This is corresponding to the descending levels of angiogenesis markers such as CD31.

Previous studies have shown that increasing BH4 synthesis can promote endothelial cell proliferation, migration and tubule formation in cultures in vitro (25). Moreover, the present study demonstrates also that $\mathrm{BH} 4$ synthesis via either the pterin salvage or the de novo pathway induces angiogenesis in tumor xenografts. These effects correlated with increases in eNOS produced NO that depended, in turn, on the activation of wild-type Ras and its downstream PI3K/Akt effectors (26). BH4 synthesis increases phosphorylation of eNOS at Ser1177 and Akt phosphorylation resulted in eNOS-derived NO rather than superoxide $(27,28)$. Theoretically, downregulation of $\mathrm{BH} 4$ synthesis could inhibit tumor angiogenesis, and GTPCH would be a therapeutic target of anti-angiogenesis for HCC. Our data suggested that downregulation BH4 synthesis via DAHP can inhibit K-ras mRNA and activation of phosphorylation of eNOS and Akt.
In the present study, we showed that in DAHP-treated tumors, BH4 and NO levels remain lower compared with the saline controls, and CD31 are lower than in controls. We suggested that there were inhibitory effects of DAHP on tumor angiogenesis, dependent on downregulation of BH4 synthesis. We found that DAHP downregulates eNOS and Akt protein expression, corresponding to decreased eNOS phosphorylation at Ser1177 and Akt phosphorylation. It is well-known that DAHP is recognized as a specific competitive inhibitor of GTPCH as it has structural similarity to GTP (29). It also acts indirectly on GTPCH by directly binding to a GTPCH feedback regulatory protein to negatively inhibit GTPCH activity (30). Our data suggested that DAHP downregulates GTPCH protein expression, with a corresponding decrease of the $\mathrm{BH} 4$ levels and contents of NO. Indeed, in the present study, decreased CD31 indicated that tumor angiogenesis was inhibited in HCC.

Taken together, our data suggested that DAHP, recognized as a specific competitive inhibitor of GTPCH, can decrease tumor BH4 and NO by the inhibition of the wild-type Ras-PI3K/Akt pathway, and then inhibiting angiogenesis. Therefore, we now give proof-of-principle that strategies targeting $\mathrm{BH} 4$ synthetic pathways may be a rational way to inhibit angiogenesis, and to control potential progression of HCC. Clearly, our recognition of DAHP in anti-angiogenesis in HCC is incomplete. In the future, we must investigate the precise mechanisms responsible for these findings to develop the most suitable treatment strategy for HCC.

\section{Acknowledgements}

The present study was supported by grants from the National Natural Science Foundation of China (no. 81201910). We thank Yao Qian for technical assistance in the experiments. 


\section{References}

1. Gacche RN and Meshram RJ: Targeting tumor micro-environment for design and development of novel anti-angiogenic agents arresting tumor growth. Prog Biophys Mol Biol 113: 333-354, 2013.

2. Stuehr DJ: Mammalian nitric oxide synthases. Biochim Biophys Acta 1411: 217-230, 1999.

3. Marinos RS, Zhang W, Wu G, Kelly KA and Meininger CJ: Tetrahydrobiopterin levels regulate endothelial cell proliferation. Am J Physiol Heart Circ Physiol 281: H482-H489, 2001.

4. Ridnour LA, Isenberg JS, Espey MG, Thomas DD, Roberts DD and Wink DA: Nitric oxide regulates angiogenesis through a functional switch involving thrombospondin-1. Proc Natl Acad Sci USA 102: 13147-13152, 2005.

5. Burke AJ, Sullivan FJ, Giles FJ and Glynn SA: The yin and yang of nitric oxide in cancer progression. Carcinogenesis 34: 503-512, 2013.

6. Fukumura D, Kashiwagi S and Jain RK: The role of nitric oxide in tumour progression. Nat Rev Cancer 6: 521-534, 2006.

7. Ying L and Hofseth LJ: An emerging role for endothelial nitric oxide synthase in chronic inflammation and cancer. Cancer Res 67: 1407-1410, 2007.

8. Barbieri A, Palma G, Rosati A, Giudice A, Falco A, Petrillo A, Petrillo M, Bimonte S, Di Benedetto M, Esposito G, et al: Role of endothelial nitric oxide synthase (eNOS) in chronic stresspromoted tumour growth. J Cell Mol Med 16: 920-926, 2012.

9. Jadeski LC and Lala PK: Nitric oxide synthase inhibition by $N^{\mathrm{G}}$-nitro-L-arginine methyl ester inhibits tumor-induced angiogenesis in mammary tumors. Am J Pathol 155: 1381-1390, 1999.

10. Katusic ZS, d'Uscio LV and Nath KA: Vascular protection by tetrahydrobiopterin: Progress and therapeutic prospects. Trends Pharmacol Sci 30: 48-54, 2009.

11. Lowndes SA, Sheldon HV, Cai S, Taylor JM and Harris AL: Copper chelator ATN-224 inhibits endothelial function by multiple mechanisms. Microvasc Res 77: 314-326, 2009.

12. Thöny B, Auerbach G and Blau N: Tetrahydrobiopterin biosynthesis, regeneration and functions. Biochem J 347: 1-16, 2000.

13. Chen L, Zeng X, Wang J, Briggs SS, O'Neill E, Li J, Leek R, Kerr DJ, Harris AL and Cai S: Roles of tetrahydrobiopterin in promoting tumor angiogenesis. Am J Pathol 177: 2671-2680, 2010.

14. Sugiyama T, Levy BD and Michel T: Tetrahydrobiopterin recycling, a key determinant of endothelial nitric-oxide synthasedependent signaling pathways in cultured vascular endothelial cells. J Biol Chem 284: 12691-12700, 2009.

15. Lampson BL, Kendall SD, Ancrile BB, Morrison MM, Shealy MJ, Barrientos KS, Crowe MS, Kashatus DF, White RR, Gurley SB, et al: Targeting eNOS in pancreatic cancer. Cancer Res 72: 4472-4482, 2012.

16. Alp NJ, Mussa S, Khoo J, Cai S, Guzik T, Jefferson A, Goh N, Rockett KA and Channon KM: Tetrahydrobiopterin-dependent preservation of nitric oxide-mediated endothelial function in diabetes by targeted transgenic GTP-cyclohydrolase I overexpression. J Clin Invest 112: 725-735, 2003.
17. Sampat KR and O'Neil B: Antiangiogenic therapies for advanced hepatocellular carcinoma. Oncologist 18: 430-438, 2013.

18. Zhu AX, Duda DG, Sahani DV and Jain RK: HCC and angiogenesis: Possible targets and future directions. Nat Rev Clin Oncol 8: 292-301, 2011.

19. Rodriguez-Viciana P and Downward J: Ras activation of phosphatidylinositol 3-kinase and Akt. Methods Enzymol 333: 37-44, 2001.

20. Engelman JA, Chen L, Tan X, Crosby K, Guimaraes AR, Upadhyay R, Maira M, McNamara K, Perera SA, Song Y, et al: Effective use of PI3K and MEK inhibitors to treat mutant Kras G12D and PIK3CA H1047R murine lung cancers. Nat Med 14: 1351-1356, 2008

21. Carmeliet P and Jain RK: Angiogenesis in cancer and other diseases. Nature 407: 249-257, 2000.

22. Stoeltzing O, Meric-Bernstam F and Ellis LM: Intracellular signaling in tumor and endothelial cells: The expected and, yet again, the unexpected. Cancer Cell 10: 89-91, 2006.

23. Phung TL, Ziv K, Dabydeen D, Eyiah-Mensah G, Riveros M, Perruzzi C, Sun J, Monahan-Earley RA, Shiojima I, Nagy JA, et al: Pathological angiogenesis is induced by sustained Akt signaling and inhibited by rapamycin. Cancer Cell 10: 159-170, 2006.

24. Zheng H, Dai T, Zhou B, Zhu J, Huang H, Wang M and Fu G: SDF-1alpha/CXCR4 decreases endothelial progenitor cells apoptosis under serum deprivation by PI3K/Akt/eNOS pathway. Atherosclerosis 201: 36-42, 2008.

25. Michaelis M, Michaelis R, Suhan T, Schmidt H, Mohamed A, Doerr HW and Cinatl J Jr: Ribavirin inhibits angiogenesis by tetrahydrobiopterin depletion. FASEB J 21: 81-87, 2007.

26. Dimmeler S, Fleming I, Fisslthaler B, Hermann C, Busse R and Zeiher AM: Activation of nitric oxide synthase in endothelial cells by Akt-dependent phosphorylation. Nature 399: 601-605, 1999.

27. Du YH, Guan YY, Alp NJ, Channon KM and Chen AF: Endothelium-specific GTP cyclohydrolase I overexpression attenuates blood pressure progression in salt-sensitive low-renin hypertension. Circulation 117: 1045-1054, 2008.

28. Ceylan-Isik AF, Guo KK, Carlson EC, Privratsky JR, Liao SJ, Cai L, Chen AF and Ren J: Metallothionein abrogates GTP cyclohydrolase I inhibition-induced cardiac contractile and morphological defects: Role of mitochondrial biogenesis. Hypertension 53: 1023-1031, 2009.

29. Kolinsky MA and Gross SS: The mechanism of potent GTP cyclohydrolase I inhibition by 2,4-diamino-6-hydroxypyrimidine: Requirement of the GTP cyclohydrolase I feedback regulatory protein. J Biol Chem 279: 40677-40682, 2004.

30. Xie L, Smith JA and Gross SS: GTP cyclohydrolase I inhibition by the prototypic inhibitor 2,4-diamino-6-hydroxypyrimidine. Mechanisms and unanticipated role of GTP cyclohydrolase I feedback regulatory protein. J Biol Chem 273: 21091-21098, 1998. 